\title{
RESUMEN DEL INFORME "UNIVERSIDAD 2000" \\ (SEGUNDA PARTE Y FINAL)
}

\section{SUMMARY OF THE REPORT "UNIVERSIDAD 2000" (SECOND AND LAST PART)}

\begin{abstract}
AUTORES
Juan José Ugalde Fernández: Profesor de Relaciones Públicas de la Facultad de Ciencias de la Información de la Universidad Complutense de Madrid (España). juan.jose@mixmail.com
\end{abstract}

\section{CURRÍCULUM VITAE}

Profesor de Relaciones Públicas de la Facultad de Ciencias de la Información de la Universidad Complutense de Madrid (España). Autor de varios artículos sobre la reforma de la ley de enseñanza superior en España, conferenciante y ponente en numerosos congresos universitarios y especialista en comunicación corporativa.

\section{RESUMEN}

Los servicios de educación, aprendizaje e investigación (objetivo de las universidades $\mathrm{y}$, en general, de las instituciones de enseñanza superior) han de proveerse de la forma más adecuada, incorporando los nuevos procedimientos que generan las tecnologías de la información y de la comunicación, lo que obliga a revisar las formas de operar de la Universidad.

\section{PALABRAS CLAVE}

Resumen - Informes - Universidad 2000 


\section{ABSTRACT}

Education services, learning and research (target universities and, in general, institutions of higher education) have to be provided in the most appropriate, incorporating new procedures that generate the information technology and communication, making it necessary to review the modus operandi of the University.

\section{KEY WORDS}

Summary - Reports - University 2000

\section{2.- Profesorado}

Interesante, aunque incompleto, análisis de los problemas del PDI. Por otra parte, de nuevo se aprovecha para cargar contra el autogobierno participativo, al mismo tiempo que se insiste en la necesidad de corresponsabilizar al personal.

Las propuestas sobre cuerpos docentes son muy incompletas. Así, no se habla del catálogo de funciones, ni por tanto las que distinguen TU de CU, con lo que también se obvia la promoción. Tampoco se justifica la supresión de TEU y CEU; por cierto, es inaceptable que en la negociación al respecto se de a los sindicatos un papel inferior al de las asociaciones corporativas.

Muy interesante la necesidad de formación docente durante la etapa de Ayudante. 
Las nuevas figuras contratadas de Titular Docente y de Titular Investigador resultan inatacables, pero debería alertarse sobre el peligro de precarización y de abuso. En particular no se concreta el tipo de contrato, aunque en (30) se dice que puede ser indefinido.

3.- Carrera académica y procedimiento de selección

Compartimos la necesidad de una carrera académica, pero las propuestas se limitan al periodo de formación y de acceso a plazas funcionarias, como ya se ha dicho. La pretensión de que la tesis doctoral sea previa a tal carrera puede frustrarse si no hay una suficiente política de becas y ayudas al respecto.

Nuevamente compartimos la preocupación por la formación docente.

Los aspectos retributivos se tocan superficialmente, como por otra parte es lógico.

Una de las mejores aportaciones es la propuesta de selección del profesorado (perfil de funciones, doble fase de preselección externa y decisión interna,...), que puede dar una salida al eterno debate al respecto. Merece nuestro apoyo total y entusiasta.

4. Personal de Administración y Servicios.

(32) No se hace ninguna alusión a las funciones de gestión y de apoyo a la docencia y a la investigación en este primer punto cuando habla de las actividades de ambos colectivos. 
(33) En este punto falta una mención en el mismo sentido al ámbito estatal que debería proporcionar esa homogeneidad entre todas las Universidades para evitar grandes diferencias entre Comunidades autónomas.

(34) El informe no hace referencia a la capacidad reconocida que tienes los Comités de Empresa para la negociación lo cual haría sumamente complicado que desde un punto de vista jurídico acuerdos de carácter autonómico se pudieran imponer sin mas a los Organos de representación de estas Universidades.

(35) Parece una contradicción que el informe recomiende el desarrollo de la carrera administrativa a la vez que defiende la figura de la libre designación como vía de acceso a los puestos de máxima responsabilidad dentro de la escala funcionarial. Además de relacionar estos puestos al servicio de las políticas universitarias lo que dista mucho del ideal de independencia que los profesionales de la gestión deberían tener. Por último la conclusión del informe instando a las Comunidades Autónomas a intensificar el ejercicio de sus competencias en materia retributiva sobre el Personal de Administración y Servicios parece un poco pobre en cuanto a su desarrollo y definición.

(36) Es interesante la necesidad de formación del Personal de Administración y Servicios, apostando por consorcios entre Universidades dentro de la Comunidad Autónoma. Un poco escaso en cuanto al desarrollo de estos planes formativos y su implicación en la carrera administrativa y laboral como vía de promoción.

\section{CALIDAD Y ACREDITACIÓN}

1.- Garantizar la calidad.

1.1. Cultura de la calidad 
1. Parece que en la actualidad ya existe una cultura de la calidad y de la necesidad de que ésta se evalúe en las universidades, y por tanto las cuestiones a plantear son los medios a aportar por la Universidad, la clase de evaluación (interna o externa), y los beneficios que se producirán.

¿Evaluar programas o evaluar instituciones? ¿Qué control o relación ha de ejercer la Administración? ¿Habilitación (conceder a la universidad el derecho de otorgar títulos) o acreditación (contrastada según criterios sobre conocimientos adquiridos o competencias profesionales)? ¿Qué grado de influencia han de tener los resultados de la evaluación en al financiación (fórmulas o contratos-programa)?

2. La evolución de la calidad permite conjugar y equilibrar a corto plazo la autonomía universitaria con la responsabilidad social.

La autonomía debe llevar consigo la responsabilidad de los órganos de gobierno y administración. Por ello, el aumento de autonomía ha traído consigo un mayor peso de las evaluaciones y de sus consecuencias.

3. El desarrollo cuantitativo, cuando ha supuesto un incremento de la calidad se ha debido a esfuerzos individuales, sin responder a coordinación o sistematización en la búsqueda de la calidad.

"Alcanzado el límite previsible de su crecimiento cuantitativo, el reto actual de la Universidad parece radicar en un esfuerzo de calidad. Es uno de los cambios mayores que deben producirse en las universidades durante el próximo decenio.

Las Administraciones Públicas y las universidades, en colaboración con otros sectores económicos y sociales partícipes de la calidad universitaria, deben proseguir 
los esfuerzos y actividades de sensibilización de la comunidad universitaria respecto a la cultura de la calidad, como elemento de equilibrio entre la autonomía universitaria y la responsabilidad social."

1.2 Preocupación por la calidad en la universidad.

4. Entre los sistemas propios actuales de calidad tenemos: de los profesores (selección, promoción y nombramiento); de los estudiantes (admisión selectiva, exámenes, becas, premios); de la investigación (financiación y ayudas, índices de citaciones y publicaciones, evaluaciones internas y externas); de los planes de estudio, cursos y enseñanza en general (evaluación y acreditación de programas y cursos, cuestionarios a los estudiantes, evaluación del profesorado, programas de formación docente); del PAS (selección y formación). Estos sistemas propios deben continuar, aunque ha cambiado el concepto de calidad, y también están cambiando los objetivos y métodos.

Acepciones de calidad:

a.- Calidad confundida con excelencia y distinción. Concepto útil para universidades de elite (en el sentido tradicional) o que intentan diferenciarse y especializarse. Inútil para un sistema universitario público. Guarda vigencia en la investigación, aunque no sea posible comparar resultados de evaluación en disciplinas o departamentos diferentes.

b.- Calidad como conformidad con unos estándares: la acreditación para otorgar títulos asegura que se cumplen unos estándares mínimos, que supera el estudiante que aprueba. 
c.- Calidad como adecuación a un objetivo o finalidad. En continuo debate. Autonomía y diversificación obligan a que cada universidad defina su propia estrategia y objetivos y mida su capacidad para cumplirlos; para ello debe desarrollar instrumentos de planificación estratégica y evaluaciones para medir la consecución de los objetivos.

d.- Calidad relacionada con eficiencia y coste de la Universidad: concepto extendido a la gestión y la administración.

e.- Calidad como aptitud para satisfacer las necesidades de los usuarios, destinatarios o clientes (industrial).

5.

f.- Calidad como capacidad de transformación y cambio (calidad total), de gestión del cambio ante las nuevas demandas de la sociedad. Los sistemas deben tomar como objetivo la mejora de la calidad.

g.- Calidad=Concepto multidimensional y complejo.

6. Funciones de los sistemas de calidad:

a.- Preparar mecanismos para la acreditación de instituciones o programas (asegurar mínimos de calidad).

b.- Mejorar la docencia, la investigación y la administración.

c.- Servir de instrumento para rendir cuentas al gobierno y a la sociedad. 
d.- Suministrar información pública y transparencia a los estudiantes y al mercado laboral (competencia y diferenciación)

e.- Operar como mecanismo para decidir una financiación diferencial de las universidades.

7. Cabe optar por un modelo de autorregulación, en que la universidad ejerza la responsabilidad en la iniciativa y ejecución, o por un modelo de control externo gubernamental, con responsabilidad en órganos ajenos. O por un modelo mixto combinando agencias independientes aprobadas por el gobierno o las propias universidades. La tendencia es combinar una primera autoevaluación interna con la evaluación externa.

2.- Experiencias de evaluación.

2.1. Experiencias en otros países.

8. "El sistema de calidad debe, por tanto, combinar el uso simultáneo de la autoevaluación interna y la evaluación externa y debe incluir la presentación pública de un informe.

9. La organización y gestión de los procesos de evaluación externa deberían encomendarse a organismos autónomos, independientes de las Administraciones y de las instituciones de enseñanza superior; pero establecidos como resultado de un acuerdo entre ambas".

Reseña de experiencias de evaluación en EE.UU., Australia, Reino Unido, Dinamarca, Francia, Holanda, Universidad de Delft, Universidad de Twente, UAB, ... 
"Existe una necesidad creciente de acreditación internacional. Para algunas universidades y para algunas titulaciones, este es un elemento fundamental para su futuro y el sistema debe facilitar este tipo alternativo de evaluación.

Las tendencias recientes marcan la aparición de redes voluntarias de acreditación entre universidades, a escala internacional. Cualquier sistema debería facilitar la existencia de acuerdos tanto a escala nacional como internacional, que permitan la colaboración de las universidades con métodos o calidad parecidos.

El sistema de calidad implantado en un sistema universitario nacional debería facilitar la transparencia y la transportabilidad hacia otros sistemas universitarios, especialmente del espacio europeo.

El sistema de calidad habría de permitir acomodar los nuevos métodos de aprendizaje a distancia y las nuevas necesidades y demandas de los estudiantes "no tradicionales". La acreditación de nuevas titulaciones y esquemas innovadores debería tener un tratamiento especial".

\subsection{Experiencias en España.}

10.11.12.13.14.15.16.17. Reseña de la evolución de los distintos planes de evaluación e iniciativas adoptadas en aras a la calidad.

Experiencias en España: plan experimental y plan nacional de evaluación de la calidad. Modelo Europeo para la Evaluación de la Calidad.

"Se necesita dar un paso más y sustituir un programa de evaluación estricto, sin consecuencias, por otro de evaluación y acreditación. No es sino la consecuencia 
lógica de una mayor desregulación del conjunto, de una mayor flexibilidad de las estructuras y de una profundización en el ejercicio de la autonomía universitaria.

Hay que advertir, sin embargo, del peligro que representa -es un punto débil de esta opción- la posibilidad de que la acreditación se transforme, con el tiempo, en un sistema normalizado (es decir, una manera de "normalización" del funcionamiento) y que a la larga cree inconvenientes a las actitudes innovadoras.

Es conveniente que los programas de evaluación actuales tengan continuidad. El balance del Plan Nacional de evaluación de la Calidad de las universidades debe servir de base para la realización de posteriores programas con objetivos más concretos y selectivos y para aumentar el grado de exigencia en los resultados."

3.- Un sistema de evaluación y acreditación.

\subsection{El sistema.}

18.19.20.21.

3.2. La calidad en el Plan Estratégico.

22. "Cada universidad debería introducir en su Plan Estratégico los mecanismos adecuados para garantizar el nivel satisfactorio de calidad en los procesos docentes y de investigación, y en la gestión."

"Los programas de calidad contenidos en el Plan Estratégico deben ser objeto de un seguimiento atento por parte de la universidad y a este respecto deben ser liderados por el rector y deben afirmar su carácter institucional abarcando la totalidad de organismos que la constituyan. De ahí su concepción transversal, que impide su 
integración jerárquica en los respectivos organigramas. Su elaboración ha de contar con la participación de todos los estamentos universitarios, para obtener el compromiso activo de docentes, estudiantes y gestores.

24. La elaboración y el desarrollo de los programas de calidad contenidos en el Plan Estratégico deben corresponder a un grupo de trabajo específico compuesto por profesores y técnicos responsable ante el rector y órganos centrales.

Alcanzado el acuerdo respecto a los programas a abordar, su prioridad y extensión, correspondería al grupo de trabajo redactar un documento detallado de las acciones a seguir, concretando sus plazos de ejecución y medios necesarios. El documento contaría con la aprobación de los órganos superiores de gobierno de la universidad. Posteriormente un informe anual daría cuenta de los avances de los programas de calidad del Plan Estratégico y de posibles medidas correctoras.

Un Consejo Asesor de Calidad -con la participación de expertos externos y profesores- formularía propuestas y asistiría al rector y al grupo de trabajo.

El Plan supone la correspondiente asignación presupuestaria y, en consecuencia, la existencia de partidas específicas para mejorar la calidad.

El objetivo último de los programas de calidad del Plan Estratégico es la mejora de la calidad. Por tanto, su credibilidad depende de conseguirla en el menor plazo posible, particularmente en la docencia. Para conseguir esta eficacia los programas que deben formar parte del Plan Estratégico exigen una cuidadosa selección, combinando el realismo con la voluntad de renovar aquellos aspectos que necesitan mejora e innovar en temas cuya futura demanda se perfila como segura."

3.3. Unidades o Consorcios de Evaluación. 
25. "De acuerdo con las recomendaciones de la Unión Europea -de modo especial, las surgidas a raíz del Proyecto Piloto Europeo de 1995- parece de una importancia capital que la organización y gestión de los proyectos de evaluación se encomiende a organismos autónomos, que gocen de independencia respecto a la Administración y las universidades en la toma de sus decisiones.

26.27.28.29 A este respecto y dadas las transferencias a las Comunidades Autónomas, el sistema de evaluación podría partir de Unidades o Consorcios de Evaluación de las Universidades radicados en los territorios de la Comunidades Autónomas, aunque sería recomendable que donde el número de instituciones sea pequeño, las Unidades pudieran abarcar instituciones universitarias que operasen en diversas Comunidades. Las Unidades deberán ser organismos autónomos dirigidos por personalidades independientes, técnicos, profesores y personas externas, nombrados por acuerdos entre la Administración autonómica -o Administraciones autonómicas, en su caso- y los órganos correspondientes de representación de las universidades en cada territorio."

\subsection{Agencia de Acreditación.}

30. "Un sistema correcto de acreditación, además de otorgar la autorización inicial para las instituciones y sus programas, debería renovar periódicamente la validez de los títulos que concede, en función de los resultados de los sucesivos programas de evaluación. La acreditación debería orientarse especialmente hacia los programas, más que a las instituciones, habida cuenta que parece haberse superado la fase meramente expansiva del sistema. No obstante, también ha de permitir, gracias a los resultados contenidos en los informes de evaluación, detectar su en alguna universidad se producen carencias graves que la puedan situar por debajo del nivel de calidad mínimo exigible en la enseñanza superior. 
En los estudios de contenido profesional se deben vincular las actuaciones de acreditación con las demandas y necesidades formativas que sugieran los distintos organismos profesionales, para proceder a su necesaria transformación".

31. "Para proceder a la acreditación se propone la creación para toda España de una Agencia de Acreditación, suficientemente alejada de los problemas y condicionantes que pueda ocasionar la proximidad de las universidades y las Administraciones Públicas. Debería, por tanto, gozar de autonomía en su actuación y libertad en la toma de decisiones como condición básica en la responsabilidad que se le encomendaría. Podría estar integrada por un número reducido de personalidades del mundo académico y profesional, nacionales o extranjeras, de reconocido prestigio y larga trayectoria en actividades y funciones relacionadas con el análisis de la calidad del sistema universitario, asistidos por un número suficiente de técnicos y personal de apoyo".

"La Agencia debería estar dedicada a las tareas de acreditación, separadas y distinguidas de los procesos de evaluación. Sin embargo, es evidente que la Agencia de Acreditación debería basar sus actuaciones en los datos resultantes de los programas de evaluación, precisándose pues la coordinación entre los dos sistemas. Sin un buen funcionamiento de los programas de evaluación institucional no podrá llevarse a buen término la iniciativa de introducir la acreditación en el sistema universitario español.

La sustitución del sistema actual "a priori" de homologación y de reconocimiento de las enseñanzas y los títulos universitarios por otro "a posteriori" como el que se propone mediante la acreditación precisaría de una revisión periódica de los estudios que se sugiere que se produzca cada 10 años. 
Los resultados de los trabajos de acreditación que realice la Agencia deben tener carácter público y los resultados han de tomar la forma de recomendaciones para las Administraciones Públicas correspondiente a quienes corresponderá tomar la decisión que proceda.

Conviene que la Agencia de Acreditación opere mediante procesos de negociación con las universidades interesadas, de modo que se establezcan plazos dentro de los cuales puedan corregirse las carencias o eliminar las debilidades que impiden la acreditación. No es la mejor fórmula que el proceso de acreditación se limite a emitir informes estrictos favorables o desfavorables -según los casos-, sin dar oportunidad a las correcciones."

32. "Por lo que afecta a la evaluación docente del profesorado los resultados no parecen satisfactorios por haberse desvirtuado el propósito inicial. Se sugiere, por tanto, proceder a un nuevo sistema de evaluación que reforme, transforme o sustituya el actual. A este respecto debería encargarse un informe que examinase lo realizado y propusiese su modificación, situándola al nivel de aceptación que ha conseguido la evaluación de la actividad investigadora del profesorado".

\section{VIII.- GOBIERNO Y ADMINISTRACIÓN}

\section{Las cuestiones}

1. Su forma de organización actual se ha de adaptar alas nuevas exigencias que el despliegue de sus funciones plantea; es decir, la Universidad ha de dirigir y administrar una organización de servicios: de educación, de investigación y otros orientados a la comunidad académica y a la propia sociedad de referencia de cada universidad. 
2. La naturaleza de los cambios que están teniendo lugar en el entorno universitario hace pensar que deberán plantearse ajustes más profundos de su estructura organizativa y de sus pautas básicas de actuación.

3. Los cambios en dicha estructura se justifican por su valor para mejorar la capacidad de la institución para resolver sus problemas.

4. No faltan motivos para que, en este tema, las cuestiones ideológicas y políticas se presenten mezcladas, de forma intrincada, con las cuestiones meramente técnicas. Hay que distinguir tres tipos de cuestiones:

Las cuestiones relativas a las relaciones entre los gobiernos y las universidades públicas.

Las cuestiones relativas al gobierno interno de las universidades.

Las cuestiones relativas a la estructura funcional de las universidades.

No existe una única combinación óptima que se pueda proponer como solución.

\subsection{Autonomía y responsabilidad social}

5. La noción de autonomía universitaria es confusa.

7. En la actualidad, la noción de autonomía universitaria se puede caracterizar en los siguientes términos:

La autonomía es una característica de la institución universitaria, no de los miembros pertenecientes a la misma.

La autonomía es una forma de organización de la Universidad que tiene una triple dimensión: la financiera, la de gestión y la académica 
8. Las universidades europeas gozan de más autonomía de gestión interna y de una cierta autonomía académica y financiera; en cambio, están más condicionadas por los gobiernos en las cuestiones relativas a la selección de personal y a la selección de los estudiantes.

9. El carácter público del servicio que prestan las universidades explica que la creciente autonomía de que gozan las universidades actualmente haya ido acompañada de una cada vez mayor demanda de responsabilidad social a las universidades.

10. Por un lado, que la organización universitaria sea capaz de suministrar información objetiva y, por otro, que el mecanismo de gobierno de la misma permita una labor de control y seguimiento.

\subsection{Gobierno interno}

11. El gobierno interno de las universidades puede tomar diferentes formas. Una primera forma de organización tradicional es la denominada forma colegial de gobierno universitario.

12. Una segunda forma corresponde a la llamada forma profesional de gobierno.

13. La forma colegial ofrece ventajas en la medida en que facilita un elevado nivel de consenso interno y garantiza una gran autonomía en el plano académico. En cambio, dificulta la gestión de la institución en diversos aspectos. La forma de organización profesional puede evitar estas dificultades, pero con la misma también se corren ciertos riesgos. 
14. En la práctica, los sistemas universitarios tienen ciertos componentes esenciales de gestión colegial, pero al mismo tiempo, se está produciendo un claro desplazamiento hacia la adopción de sistemas más profesionalizados.

\subsection{Organización disciplinar y organización por funciones}

15. Tradicionalmente la organización de las universidades ha respondido a las grandes líneas de división de las disciplinas académicas.

16. Varios factores están contribuyendo a diluir esta estructura tradicional. La diversificación de los curricula académicos ha roto la anterior correspondencia entre éstos, las facultades tradicionales y los títulos profesionales.

17. La organización resultante se acerca, cada vez más, a lo que se conoce como un modelo de organización matricial. El modelo matricial es en principio más flexible y eficiente que el tradicional, incorpora más rápidamente a la estructura universitaria las modificaciones que se producen en los diferentes ámbitos científicos. La organización matricial favorece un modelo de gestión más profesional que colegial.

Los aspectos negativos son que aumenta la complejidad organizativa, produce interferencias entre los diferentes órganos de gestión y existen solapamientos

18. La visión de una organización de prestación de servicios públicos empieza a dibujarse en la organización de la Universidad. Los receptores de los servicios universitarios están pidiendo su cuota de poder.

19. La Universidad adopta, progresivamente, distintos esquemas organizativos e incluso nuevas estructuras jurídicas para gestionar los nuevos servicios. La 
organización universitaria ha de proceder a seleccionar aquellos servicios y aquellos ámbitos en los que quiere estar presente.

2.-Modelos de organización universitaria

\subsection{Modelos abstractos}

20. Se pueden distinguir hasta tres modelos de gestión universitaria: el modelo burocrático, el modelo colegial y el modelo de mercado.

21. Los sistemas de gestión burocráticos están regidos por el derecho administrativo por las pautas de comportamiento características de las administraciones públicas, como en Francia y en los países del Sur de Europa. Se acepta que este modelo es demasiado rígido para la gestión de las universidades y que restringe su capacidad de responder a los cambios en su entorno social.

22. En el modelo colegial la Universidad es considerada como una entidad completamente autónoma, con normas propias de funcionamiento con un papel muy preponderante a la comunidad académica. Tiene el riesgo de inclinarse excesivamente hacia el inmovilismo y el corporativismo, limitar la capacidad de respuesta a los nuevos retos.

23. El modelo empresarial o de mercado parece el sistema más compatible con el de las universidades de titularidad privada. La ventaja de este sistema residen en su flexibilidad y en su capacidad de respuesta organizativa a los nuevos retos de las universidades. Los problemas, en cambio, provienen del escaso margen de autonomía académica que el mismo garantice. 
24. Los tres modelos pueden considerarse como tres tipos ideales situados en los vértices de un triángulo cuyo interior define el espacio real de posibilidades

25. Se detectan ciertas tendencias de carácter general. Se puede caracterizar en:

Aumento de la autonomía institucional correlativa a un aumento del control social sobre las universidades.

Profesionalización de la gestión interna de las universidades.

Potenciando la diversificación y la competencia entre universidades.

2.2 La situación del sistema universitario español

26. La LRU implantó un modelo híbrido de coordinación y de gestión de la Universidad. Otro de los objetivos era democratizar el sistema universitario y dar un fuerte impulso a los objetivos de modernización de la Universidad.

27. La solución organizativa que cristalizó finalmente no responde a ninguno de los tres modelos apuntados anteriormente. Las universidades son financiadas en su mayor parte por la Administración Pública, pero ésta no interviene en el gobierno interno de la institución. La gestión de las universidades se rige por las normas del derecho administrativo, aunque, gocen de considerable autonomía organizativa y presupuestaria. En ocasiones, la autonomía universitaria ha derivado hacia un corporativismo académico, a menudo de miras sumamente estrechas. El modelo de la LRU se aproxima al modelo colegial, desplazándose muy levemente hacia el modelo de gestión profesional y técnico.

3.- La primacía de las competencias y de los procesos académicos

3.1 Significado de los distintos procesos 
28. Es necesario buscar un cierto equilibrio entre la capacidad de la oferta universitaria, las expectativas de la demanda de la sociedad y las políticas e iniciativas de los reguladores.

29. La capacidad de supervivencia de la Universidad deberá sustentarse en las competencias académicas y en los procesos académicos clave

30. Por procesos académicos clave se entiende el conjunto de actividades que permiten llevar a cabo la misión docente e investigadora de la Universidad.

32. Para conseguir el cumplimiento de su misión y de sus objetivos, la Universidad ha de estar en condiciones de controlar un conjunto de procesos - académicos, de apoyo y de gobierno-

\subsection{Procesos académicos: estudios}

33. La eficaz gestión de las funciones de tipo transversal es una de las debilidades reconocidas del actual sistema de organización de las universidades.

34. Se aconseja establecer una organización académica responsable de gestionar la primera etapa de las enseñanzas de tipo A que no se fundamente en la estructura habitual por departamentos.

35. Los estudios disciplinares del segundo tramo de las enseñanzas del tipo A y del tipo C pueden ser gestionados correctamente con una estructura organizativa más tradicional, basada en los departamentos. La caracterización de los estudios de predominio del output parece aconsejar una gestión por unidades que los organice de forma integrada en función precisamente de dicha salida profesional. 


\subsection{Procesos académicos: investigación}

37. Las actividades de investigación plantean una gran variedad de tipologías de organización, desde el investigador que trabaja individualmente hasta los grandes centros de investigación públicos o en colaboración con la iniciativa privada.

38. La Universidad española debería, por tanto, dar apoyo a esta estructura multinuclear y con distinto nivel de formación en su organización. Sin embargo, la tendencia prioritaria se ha de encaminar hacia la consolidación de grupos de excelencia en investigación. La creación de unidades de gestión y de apoyo en la unidad para cualquiera de los niveles mencionados es, indispensable para el adecuado funcionamiento profesional de la investigación.

\subsection{La gestión de los procesos académicos}

40. El diseño de planes incluye el desarrollo y mejora de los planes de estudio, de las líneas de investigación y del desarrollo de los demás servicios a la sociedad. Es uno de los procesos claramente mejorables en las universidades y las actividades de promoción de estudiantes, de captación y movilización de recursos, para la investigación y para las demás actividades de la universidad, van a requerir un mayor impulso dado el entorno más competitivo que parece que se dibuja para las universidades.

43. Para la gestión de los procesos académicos se propone, en primer lugar, dar mayor importancia a los órganos ejecutivos que se responsabilizan de su gestión. Bastaría un refuerzo de las estructuras administrativas de los departamentos y una integración de algunos de ellos según grandes áreas del conocimiento científico. Una segunda recomendación consiste en diseñar distintas formas de organización para la 
prestación de los distintos servicios universitarios, logrando así, una mayor flexibilidad organizativa.

4.- Funcionalidad de los procesos de gobierno

4.1 Elaboración y desarrollo de la estrategia: planificación estratégica

44. La organización y la gestión de los procesos de cada universidad forman parte de las funciones de los órganos de gobierno de la misma y están estrechamente relacionados con la configuración de su plan estratégico y de la definición de su misión. El plan estratégico propio de cada universidad, debe:

conocer el grado de satisfacción de los diferentes destinatarios de los servicios que presta la Universidad.

desarrollar la visión y la estrategia de la institución.

La fijación clara y explícita de los objetivos de la Universidad, es indispensable también para la rendición de cuentas de sus resultados y par reclamar los recursos públicos que precisa. El desarrollo de un programa de comunicación y de relaciones públicas, se convierte pues en un elemento fundamental para llevar a buen término nuevas iniciativas y actividades.

\subsection{Estructura de gobierno}

45. El déficit de eficiencia en la gestión proviene fundamentalmente de la intrincada red de órganos colegiados, de las limitaciones que los sistemas de elección y control imponen a los órganos unipersonales, y de la complejidad de los procedimientos de participación en la toma de decisiones. 
La estructura de gobierno debería orientarse claramente hacia un modelo más profesional, que potenciase la flexibilidad y la eficacia en la gestión y facilitase la rendición de cuentas a la sociedad.

46. Se sugiere, en primer lugar, mantener como autoridad máxima de la Universidad la figura del Rector. La Junta o Consejo de Gobierno sería el órgano máximo de gobierno de la Universidad. De ella formarían parte, además del Rector, el Presidente del Consejo Social y el Gerente y un número reducido de miembros, más de la mitad deberían ser representantes de la comunidad universitaria, elegidos por el Claustro, y el resto de personas de comprobada capacidad de gestión y representación social, elegidas por el Consejo Social.

47. El tamaño del Claustro debería reducirse para hacerlo más operativo. El presidente del Consejo Social podría ser nombrado directamente por el Gobierno de la Comunidad Autónoma correspondiente.

48. El Rector podría nombrar un equipo de carácter ejecutivo. Además de estos órganos de gobierno es preciso articular todo un sistema de administración descentralizada. El equilibrio podría conseguirse, por ejemplo, haciendo que los responsables de procesos orientados directamente a prestar servicios "hacia el exterior" dependieran de la Junta de Gobierno mientras los responsables de procesos más orientados "hacia dentro" fueran elegidos por los consejos correspondientes.

5.- Carácter instrumental de los procesos de apoyo

\subsection{Los procesos de apoyo}

49. La mayoría de estos procesos suelen entrar dentro del ámbito tradicional de responsabilidad de la gerencia universitaria. 
5.2 Gestión de recursos financieros y materiales

50. Es indispensable que las universidades identifiquen los productos y servicios que ofrecen y desarrollen los instrumentos de contabilidad analítica, que permitan conocer sus costes.

51. La identificación de las unidades de coste y la imputación de los costes de los servicios de la universidad responsabiliza a las distintas unidades en la toma de decisiones. El presupuesto de la universidad debería convertirse en el mecanismo para distribuir los fondos de acuerdo con la planificación estratégica formulada por sus unidades docentes y de investigación.

54. Las universidades tratan de lograr una mayor financiación tanto de sus servicios tradicionales como de nuevos según estrategias no uniformes que tratan de responder a los cambios del entorno.

55. La búsqueda de estas nuevas fuentes de financiación puede hacerse de tres formas:

Una primera forma supone conseguir nuevos ingresos en los servicios tradicionales de la Universidad a través del cobro de prestaciones por:

Servicios prestados.

Explotación externa y venta de productos.

Uso de las instalaciones.

Publicidad en publicaciones y en otros soportes.

Un segundo camino para conseguir nuevos ingresos se basa en la obtención de fondos adicionales para afrontar las actividades habituales de las universidades. 
Finalmente, la tercera forma se orienta a conseguir fondos para promover nuevas actividades, algunas de las cuales derivan de las oportunidades abiertas por la fase actual de cambio tecnológico. Financiar los proyectos innovadores es uno de los problemas básicos a los que se enfrenta cualquier institución que desee adaptarse al cambio. La financiación inicial podría proceder de la propia universidad o combinar los programas de subvenciones y de ayudas públicas y con ayudas privadas, y no hay que descartar el acudir directamente a fondos procedentes del sector privado.

56. Las universidades deben dotarse de una contabilidad patrimonial, actualizando con ello sus activos patrimoniales - construcciones, equipamientos e instalaciones -, que permita contabilizar el monto anual que ha de dedicarse a amortizaciones.

57. En los últimos años se ha ampliado el abanico de nuevos servicios reforzando el carácter de la universidad como proveedora de servicios a los estudiantes y al resto de la sociedad. La definición, contenido y función que se asigne a los nuevos servicios universitarios será un elemento fundamental que definirá la estrategia general de la Universidad y será elemento diferenciador.

IX.- REDES TECNOLOGICAS Y REDES UNIVERSITARIAS

\section{(A) RESUMEN Y PROPUESTAS}

1.- Introducción de las TIC en la Universidad

1.1.- Las tecnologías de la información y comunicación (TIC)

(1) Las TIC han experimentado un desarrollo espectacular, y en el futuro inmediato afectarán fuertemente a la U., 
(2) tanto por cuestiones de demanda

(3) como de oferta (docencia, investigación, administración)

(4) Sin embargo, las U. han respondido de forma desigual

(5) mientras que los nuevos proveedores de educación superior suelen utilizarlas de forma intensiva.

1.2.- Incorporación de las TIC en las U.

(6) Las principales dificultades para la introducción de las TIC en las U. son: falta de estrategia institucional, resistencia a usarlas en docencia, costos elevados.

Se aconseja que las U. entren en la educación a distancia, con una cierta dosis de docencia virtual.

(7) Las U. deben clarificar su decisión estratégica sobre el uso de las TIC en docencia y demás servicios. En particular: dosis de docencia virtual, nuevos colectivos de estudiantes, formación continuada,...

(8) La inversión en TIC fracasará si el profesorado no está motivado: incentivos para formación en TIC, reversión de ingresos hacia el propio centro o departamento,... Aunque el profesor no vaya a resultar substituido, será menos emisor y más tutor, con una parte de enseñanza no presencial.

(9) Para hacer frente a las inversiones y a la competencia, las U. deberán colaborar con entidades externas (de telecomunicaciones, información, ocio,...), con otras U. 
(material docente) y con agentes económicos, sociales y profesionales (diseño de formación continuada,...).

(10) Para cubrir los elevados costos de las TIC habrá que acudir a aportaciones externas, pero sobre todo a redistribuciones internas de los fondos, tendiendo a constituir un fondo estable y suficiente.

(11) Hay que programar tanto las inversiones y renovaciones (alta obsolescencia), como el personal (asesoramiento técnico, generación de productos educativos,...)

(12) Igualmente, nuevas unidades de apoyo para ayudar al profesorado a incorporar las TIC.

(13) Las U. han de introducir la reingeniería de procesos administrativos (consulta del propio expediente, tasa de éxito,...) y la gestión por proyectos de la docencia que requiere para cada asignatura o curso: presupuesto (estudiantes, objetivos, clase de tecnología,...), propuesta de equipo (docentes, diseñadores, técnicos TIC,...), calendario,,..

(14) Las U. han de facilitar el acceso de los estudiantes a las TIC (ordenadores, red,...). Las aulas informáticas han resultados muy costosos, por lo que se tiende a que cada estudiante disponga de su propio ordenador, desarrollando ayudas al efecto (becas, donaciones,,..$)$

(15) Las U. han de fortalecer los servicios de sus campus: la integración en el medio universitario consigue un desarrollo personal mucho más rico que la enseñanza virtual. Las actividades fuera del aula y del laboratorio probablemente determinarán las preferencias de los estudiantes. 
2.- Formas de cooperación entre universidades

2.1.- Redes universitarias.

(16) Se pone de relieve la necesidad creciente de cooperación interuniversitaria en forma de red: mundialización de la economía, factor positivo para la localización de empresas, dinamización económica del territorio, mercado de trabajo continental, multidisciplinariedad (temas medioambientales,...), obtención de fondos,... Las TIC lo facilitan.

(17) Se entiende por "red" una forma de colaboración no jerárquica entre U., o entre éstas y la Administración pública y el sector productivo. Entre sus ventajas: flexibilizar estructuras, comunidad de acción, mayor número de unidades implicadas,,..

(18) Las motivaciones principales han sido:

intercambio de información

movilidad de estudiantes y profesores

potenciación de la especialización y de las franquicias

estándares comunes de calidad

provisión de servicios complejos

internacionalización de los curricula

titulaciones y doctorados compartidos

intercomunicación de investigaciones

formación continuada

(19) Entre los requisitos para el éxito:

liderazgo 
toma de decisiones (a nivel central y de cada unidad)

confianza mutua

motivación de las personas implicadas

masa crítica

relaciones a largo plazo

definición y coordinación de objetivos

preservación de la identidad de cada U.

operatividad

La financiación privada no ha de comprometer la independencia de la U., ya que presta un servicio público.

2.2.- División del trabajo ente U.

(20) La cooperación entre instituciones requiere una cierta división del trabajo: la red proporciona un marco estable. La cooperación se ha convertido en una dimensión básica de la estrategia de la U., sobre todo la intraeuropea.

Tres tipos de organizaciones internacionales:

asociaciones académicas: académicos y sus unidades

consorcios académicos: agrupaciones de unidades académicas para ejecutar un contrato determinado, habitualmente financiado externamente

redes institucionales: grupo de instituciones (U., empresas, administraciones públicas) con objetivos estables y no perecederos (movilidad, cooperación en investigación,...)

(21) Pueden surgir por:

respuesta a una demanda exterior

centros de excelencia (docente o investigadora) 
deseos de colaboración surgidos internamente acceder a programas concebidos por terceros ideales de solidaridad

Cada U. ha de ir asumiendo la financiación, substituyendo la exterior inicial.

No deberían circunscribirse al ámbito europeo.

2.3.- Alianzas estratégicas de la U.

(22) La competitividad empresarial se ha acentuado, obligando a elecciones estratégicas para concentrarse en ámbitos concretos, externalizando otros. Por ello han crecido las redes de cooperación (horizontales o verticales; de naturaleza jerárquica o coordinada; informales o normativizadas) entre ellas.

También entre organizaciones culturales, sanitarias, sociales,...

(23) Las alianzas estratégicas ente U. y otras organizaciones requieren una mayor concreción. No como un mero contrato de investigación, sino precisando:

congruencia de objetivos

reparto de presupuestos

oficinas de enlace (habitualmente el rectorado)

metodología y estándares de calidad

derechos de propiedad intelectual

solución de discrepancias

formas de abandono

Las alianzas estratégicas ayudan a la financiación y a la autonomía. No debería ser una sola, sino varias convenientemente coordinadas. 


\section{4.- Colaboración territorial.}

(24) En España, la expansión territorial ha sido influida más por intereses locales que por programación racional, promoviendo más la confrontación que la competencia. Debería atenderse a:

colaboración y coherencia del sistema

equilibrio territorial y social

diversidad de la oferta

suficiencia financiera de cada $\mathrm{U}$.

(25) Dos modelos:

polarización en grandes concentraciones urbanas

redes en ciudades pequeñas y medianas

La política universitaria debe aprovechar las ventajas de ambos.

(26) La cooperación territorial requiere órganos de coordinación, representativos de las U. y de la sociedad, paralelos a la Administración Pública territorial.

(27) La mayor autonomía universitaria requiere mayor eficiencia y mayor responsabilidad.

Las U. de gran tamaño deberían descentralizarse en un "sistema" o dividirse.

En un "sistema" las funciones de los órganos centrales suelen ser:

asegurar la debida excelencia 
adaptar el flujo de recursos

reservar el carácter comprensivo

cuidar que la competencia resulte beneficiosa

cooperar con el resto de instituciones

(B) VALORACION Y SUGERENCIAS

\section{1.- Las TIC}

Quizá se exagera la trascendencia de las TIC. En especial la docencia virtual y semipresencial. En ese sentido, hay que evitar la imagen de que la docencia presencial es algo obsoleto (ni siquiera la de tiza y pizarra). Igualmente, hay que evitar la hipertrofia de los órganos promotores, incentivos, etc. de las TIC.

Pero es cierto el peligro de que la U. pública pueda aparecer como desfasada: se requiere una decisión estratégica al respecto.

Acertada alerta sobre los costos de implantación y mantenimiento de las TIC. Incluso pueden obligar a cofinanciación, que comprometa la independencia de la U.

También acertada alerta de que el estudiante virtual se pierde gran parte de los valores de la formación universitaria.

\section{2.- Cooperación entre U.}

Importante defensa de la cooperación (redes, alianzas estratégicas) entre U., aunque paradójicamente algunos argumentos son mercantilistas (para mejor afrontar la competencia). Habría que insistir en los argumentos ideológicos de la cooperación: servicio público, progresión del conocimiento, el conocimiento al alcance de todos, etc. 
En cuanto a las redes y alianzas con sectores privados, es muy dudoso que sea compatible con la autonomía y la independencia de la U. Se dice que hay que evitar ese peligro, y propone para ello la multiplicidad de alianzas; pero no parece antídoto viable ni suficiente. Por tanto, las alianzas estratégicas habría que limitarlas al sector público.

Totalmente de acuerdo con el apartado sobre política territorial, aunque debería desarrollarse más e primer párrafo. Concretamente debería plantearse claramente la alternativa entre el modelo de "especialización y cooperación planificada" frente al "competitivo de confrontación de intereses", y argumentar más detalladamente en favor del primero.

\section{ANEXO}

Este apartado se ha elaborado en la Secretaría de Universidad con la intención de sugerir su inclusión el en capitulo VI apartado 4-(36) del Inform Bricall.

\section{APORTACIONES PARA EL PERSONAL DE ADMINISTRACIÓN Y SERVICIOS}

Los años ochenta trajeron consigo un aumento muy importante del número de estudiantes universitarios en tal cantidad que desbordó la capacidad de recepción y atención cualificada por parte del personal encargado de la administración y los servicios universitarios, tanto en lo que hacía referencia a las tareas de apoyo a la docencia y a la investigación como a las tareas administrativas y de gestión.

Eran los años donde un Convenio Colectivo único para el personal laboral y la dependencia directa del Ministerio de Educación -Organismos Autónomos- para todo el funcionariado, ayudó a construir el concepto de que el PAS podría tener una 
entidad propia, definida y diferenciada del resto de las personas que le trabajaban a las distintas administraciones públicas, especialmente a las administraciones educativas.

Se promueven en esa década diversos encuentros estatales para el PAS de las Universidades con apoyos económicos de los equipos gerenciales de aquellos años, con el intento de fructificar en un sindicalismo de tipo asambleario o de corte corporativo, pero se encontraron con una apuesta decidida de los sindicatos más representativos que hizo fracasar aquellas tentativas y que en definitiva acabó siguiendo los pasos de la inmensa mayoría del personal laboral y funcionario de las administraciones. No obstante, se consiguió crear un poso por el que se concibió el PAS-Universitario como un conjunto global con dos únicas y claras diferencias: Personal laboral para los servicios y personal funcionario para la administración.

Las transferencias educativas a las comunidades autónomas con competencias plenas: Euskadi y Navarra, Catalunya, Galicia, País Valencià, Andalucía y Canarias van a provocar una fuerte distorsión y generarán nuevas tensiones alimentadas por las tendencias centrífugas que provocaran el nacimiento de nuevos convenios colectivos autonómicos e incluso de empresa.

La creación de nuevas universidades fue el segundo paso después de la aprobación de los estatutos de las Universidades, uno y otro crearon condiciones para refugiarse más en lo local y en lo más próximo, de este modo se generaron las condiciones de una nueva concepción, fines, actividades y especialización del PAS.

Las oleadas liberales en la concepción de la política, especialmente en la política universitaria sobre al gestión de los recursos humanos, introducen el elemento de la privatización en la prestación de servicios universitarios, pero planteados siempre como procesos de externalización para evitar el conflicto directo con los sindicatos. 
Paso a paso los gestores universitarios superaron sin problemas la puesta en marcha de un proceso -privatizador- que se ha desarrollado sin conflicto es más, en muchas ocasiones con propuestas muy solventes para el personal propio de la Universidad de tal modo que ha resultado incluso atractivo. En definitiva, se consolida un nuevo tipo de PAS continuista con las relaciones anteriores, personal estable en una gran mayoría a través de un régimen laboral o funcionarial. Pero también nace otro PAS con una relación indirecta con la Universidad, establecida ésta a través de contratas de servicios, subcontratas, sociedades propias y mixtas, fundaciones, etc...

Con todo el PAS fue encontrando un espacio propio cubriendo la prestación de servicios universitarios y dedicándose a la administración de las acciones universitarias. La creación de nuevas universidades desde la década de los ochenta ha incrementado notablemente el peso del PAS y la necesidad creciente de su especialización que han venido a resaltar su papel e importancia en el apoyo a la investigación y a la docencia, así como en tareas no solo administrativas sino también en la gestión económica, tecnológica y científica.

La gestión de la internacionalización lleva consigo una pesada carga administrativa que las universidades deben asumir. El seguimiento de los proyectos internacionales, tanto en su faceta de movilidad de personas como las facetas de investigación requieren tiempo, esfuerzo y la participación del PAS especializado en la contabilidad y en el control, que no siempre están disponibles en los departamentos universitarios y que deben ser claramente diferenciados de las tareas del profesorado.

Desde el punto de vista expuesto se refuerza la propuesta de separar las funciones docentes y de gestión, dedicándose los profesores a las primeras y coordinándose las segundas con personal especializado. Debemos redefinir pues al PAS-Universitario para encajar tanto al personal más directamente implicado en servicios de apoyo a la 
investigación y en la gestión económica y administrativa, con también al amplísimo colectivo de PAS con tareas auxiliares, compartimentadas y muy especializadas que trabajan en los campus universitarios. El uso de las nuevas tecnologías, la internacionalización de las actividades universitarias y la imperiosa especialización en la administración y en la gestión van a condicionar un nuevo concepto y nuevos cometidos para un el PAS universitario. Una apuesta decidida por la calidad de todos estos servicios debe imponerse para romper las tendencias actuales que pretenden desvincular de la Universidad al personal que realiza tareas auxiliares mediante formulas de relación laboral específicas y extraordinarias, este es el reto al que nos enfrentamos para el inmediato futuro y frente al que debemos plantearnos alternativas para alcanzar la excelencia universitaria y hacercarnos a sociedades más regularizadas y estables.

Las ratios PAS/PDI nos indicaran el coste del PAS en función de su relación con el PDI. Este es uno de los aspectos más difíciles de abordar debido a la falta de definición, todavía, en el concepto del PAS ¿qué incluimos?. A nadie escapa que en las universidades norteamericanas uno de los índices de calidad que se tienen muy en cuenta es el factor de la ratio PAS/PDI. La relación 1x1 es el estandar de calidad norteamericano para especialistas en los análisis de valoración de calidad de las universidades. La repercusión sobre el coste total del sistema universitario no es en absoluto despreciable cuando nos referimos a valores de gasto entre el 17\% y el 20\% de los costes de personal en los que se mueven las universidades españolas contabilizando solo al personal contratado directamente por las universidades. Pues bien, el Informe Universidad 2000 adolece de estadístisticas, análisis y estudios que refuten o sostengan afirmaciones en uno $\mathrm{u}$ otro sentido. Un dato, en las universidades valencianas, según el Libro Blanco del sistema Universitario Valenciano nuestra relación PDI/PAS se sitúa en el 2,2 mientras que el coste medio de un PAS se calcula en torno a los 3.300 .000 pesetas. Para abordar con más rigurosidad este apartado que ha sido olvidada en el Informe Universidad 2000 
deberían incorporar los estudios realizados al respecto para establecer unas reflexiones más precisas y poder formular alternativas adecuadas.

\section{FILOSOFIA DEL PERSONAL DE GESTIÓN}

1.- El PAS como gestor de la Universidad.

La universidad es un organismo público que debe dar respuesta a las necesidades de colectivos de usuarios muy diversos, todo ello en una organización caracterizada por la coexistencia de numerosos centros de poder. (Centros de poder que son tanto puramente políticos "MEC, CC.AA"., como politico-académicos," Rectores, gerentes, decanos", como corporativos.)

La creciente complejidad de la gestión, hace necesaria la presencia de personal que haga funcionar el sistema, poniéndolo al servicio de las actividades propias de la universidad. Colaborando, en suma, en la buena marcha de los programas científicos y académicos y en el buen funcionamiento de la administración de la universidad.

La gestión universitaria puede ser eficiente si es rigurosa a imaginativa, sin caer en la trampa del mimetismo con la gestión privada, porque tiene y ha de tener un estilo propio, al que son inherentes la democracia interna y la participación, que se han de hacer compatibles con la agilidad y que permiten movilizar un caudal importante de voluntad y de inteligencia, los dos recursos menos escasos, probablemente, en la universidad pública.

2.- Apoyo a la docencia y la investigación.

Como institución dedicada a la investigación científica y a la educación superior, la universidad se caracterizaría en nuestros días por ser, simultáneamente, una empresa 
pública de investigación científica, un servicio público de educación superior, y una empresa pública de información científica y cultural.

Se podría proponer una plantilla integrada, con puestos de trabajo bien definidos, con independencia de la relación administrativa o laboral de las personas que los ocupen.

Cada puesto de trabajo debería tener definidas sus características, requisitos de acceso y funciones, así como sus relaciones orgánicas y funcionales.

El sistema de gestión contaría con tres tipos de personal:

Personal técnico: ejerce las funciones específicas de un servicio.

Personal auxiliar especializado: no realiza tareas técnicas, pero sí tareas de apoyo para las que se requiere una cierta formación.

Personal de administración: ejerce las funciones propiamente administrativas como institución.

Por otra parte, cada universidad debería elaborar un programa de formación y reciclaje del personal que permitiera una actualización continua de los conocimientos necesarios para el ejercicio de la profesión.

En conclusión, el personal de gestión debe ser, desde su posición de apoyo a la docencia, a la investigación y a la propia gestión administrativa de la universidad, el órgano encargado de obtener el máximo rendimiento social de las inversiones públicas en el sistema público universitario, de la investigación y de la enseñanza superior y sin estar sujetos a tendencias o criterios políticos concurrentes.

4.3. Carrera administrativa y carrera laboral 
1. El nombre de carrera administrativa indica el itinerario individual de progresiva capacitación y adquisición de competencias que adquiere el trabajador desde su ingreso en los niveles básicos de la administración universitaria, hasta los más altos niveles administrativos y de gestión. El mismo esquema es aplicable al Personal Laboral en cuanto a sus competencias desde grupos y categorías bases hasta niveles de apoyo docente e investigador del mas alto nivel. Si bien no hay que obviar la posibilidad de acceder a puestos de categorías intermedias o altas directamente cuando se dan ciertos requisitos tales como titulación o superación de pruebas de acceso especificas.

Actualmente se hace difícil reconocer una autentica carrera administrativa dentro del Personal de Administración y Servicios debido entre otras cosas a los malos diseños y carencias de los diseños de plantillas, así como por la utilización, en algunos casos indiscriminada, de figuras como los puestos de libre designación.

2. Un modelo de carrera administrativa o laboral debería establecer claramente cuales son los niveles básicos de formación y las exigencias mínimas para la promoción hacia niveles o categorías superiores. Igualmente debería evitar situaciones de alta precariedad o contratación que ejercen una fuerza enorme sobre la promoción al tener que adaptar el sistema a la estabilización del Personal de Administración y Servicios.

En la mayoría de Universidades el acceso es vía concurso-oposición pero se constata una contratación temporal muy alta como vía de ingreso a la Universidad, esta contratación masiva genera muchas dificultades a la hora de establecer una autentica promoción profesional además se observa un taponamiento muy elevado al llegar a los niveles y categorías intermedios debido a la contratación y los puestos de libre designación en niveles superiores. 
3. La inclusión por tanto de estos niveles de gestión y apoyo a la docencia e investigación dentro de las RPT así como la reducción de la precariedad se hace fundamental como primera medida para diseñar la carrera administrativa. Potenciar la promoción a través de la formación continuada como principal elemento a valorar debería ser el camino a seguir a semejanza del modelo de carrera académica que se pretende instaurar para el profesorado universitario.

4. La compatibilidad entre las diferentes RPT es fundamental a la hora de elaborar las futuras plantillas para evitar una deshomogeneización que llevaría a hacer imposible la movilidad del Personal de Administración y Servicios entre las diferentes Universidades. En este sentido la vía para armonizar las RPT sería un acuerdo dentro del marco del Protocolo de Negociación en el ámbito estatal para el Personal de Administración y Servicios de las Universidades Publicas Españolas.

\section{ESTUDIO LEGISLATIVO SOBRE EL PERSONAL DE ADMINISTRACIÓN Y SERVICIOS DE UNIVERSIDAD}

Existen distintos ámbitos legales:

Ley de medidas para la Reforma de la Función Pública (30/84)

Legislación de función pública de las Comunidades Autónomas y normativa concordante.

Ley orgánica 11/83 de Reforma Universitaria

Normativa de transferencias en materia de educación universitaria de las Comunidades Autónomas.

Estatutos específicos de cada una de las Universidades

Convenios colectivos de ámbito autonómico o estatal para el Personal Laboral. No aparece ninguna referencia de los ámbitos funcionales del PAS hasta llegar a los estatutos específicos de Universidad. 
En la perspectiva de una eventual modificación de la Ley Orgánica 11/83 de Reforma Universitaria estimamos conveniente la modificación de la denominación del Título VI como "Del Personal de Gestión, Administración y Servicios" y la adición de un punto 5 en el artículo 49 del mismo Título VI en la que se enunciaran con carácter general los ámbitos funcionales de este personal incidiendo en los aspectos de gestión en sus diversas áreas y la de soporte a la actividad docente e investigadora.

Se propone un avance de redacción del punto 5:

"Serán, con carácter general, funciones del Personal de Gestión, Administración y Servicios las de soporte para la actividad docente e investigadora. Así mismo es competencia de este personal el desarrollo de las labores de gestión administrativa, económica, académica, de servicios de biblioteca, informática, información y cualquiera otro que se determine necesario por la Universidad para el cumplimiento de sus objetivos." 